\title{
Optimization on submanifolds of $\delta$-Lorentzian trans-Sasakian manifolds with Casorati curvatures
}

\author{
Aliya Naaz Siddiqui, Mohd. Danish Siddiqi and Mohammad Hasan Shahid
}

\begin{abstract}
The present research article is concerned about a couple of optimal inequalities for submanifolds of $\delta$-Lorentzian trans-Sasakian manifolds endowed with semi-symmetric metric connection (briefly says $S S M$ ). Some examples of $\delta$-Lorentzian trans-Sasakiam manifolds are also discussed here. This paper ends with some open problems.
\end{abstract}

Keywords. $\delta$-Lorentzian trans-Sasakian manifold, semi-symmetric metric connection, Casorati curvatures.

\section{Introduction}

The theory of differentiable manifolds with Lorentzian metric is an interesting topic in differential geometry. Matsumoto [17] popularized the knowledge of Lorentzian para-contact manifolds. In [8] CR-Submanifolds of trans Lorentzian para Sasakian manifolds have been investigated by Gill and Dubey. In [23], Pujar and Khairnar have discussed some axioms of the Lorentzian transSasakian manifolds and studied the some basic results. In fact, if $\overline{\mathbf{M}}$ has a Lorentzian metric $g$, that is a symmetric non-degenerate $(0,2)$ tensor field of index 1, then $\overline{\mathbf{M}}$ is known as a Lorentzian manifold. Since the Lorentzian metric is of index 1, Lorentzian manifold $\overline{\mathbf{M}}$ has exhibits three kind of vector fields

1. spacelike vector fields,

2. timelike vector fields, and

3. lightlike vector fields.

This is the major difference with the Riemannian case gives interesting properties on the Lorentzian manifold. A differentiable manifold $\overline{\mathbf{M}}$ has a Lorentzian metric if and only if $\overline{\mathbf{M}}$ has a 1- dimensional distribution. Both odd and even dimensional manifolds are able to have a Lorentzian metric. Motivated by the above researches and remarks Bhati [1] developed the conception of $\delta$-Lorentzian trans-Sasakian manifolds. Follow by Siddiqi et al. [12] who studied the contact $C R$-submanifold of a $\delta$-Lorentzian trans-Sasakian manifold.

Received date: January 4, 2021; Published online: May 1, 2021.

2010 Mathematics Subject Classification. 53C15, 53C25.

Corresponding author: Mohd. Danish Siddiqi. 
Friedmann and Schouten [7] came up with the central idea of semi-symmetric linear connection on a differentiable manifold in 1924. Later on, Bartolotti [2] gave a geometrical meaning of such a connection and then Hayden [10] devolved the discourse of semi-symmetric metric connection. In [29], Yano further enhanced the systematic study of this connection on a Riemannian manifold. Therefore, several geometers studied this connection on different manifolds and obtained very interesting results (for example, [11], [13], [22], [24], [25]).

Let $\nabla$ be a linear connection in an $m$-dimensional differentiable manifold $\overline{\mathbf{M}}$. The torsion tensor $\mathcal{T}$ and the curvature tensor $R$ of $\nabla$ are given respectively by

$$
\mathcal{T}(E, F)=\nabla_{E} F-\nabla_{F} E-[E, F]
$$

and

$$
R(E, F) G=\nabla_{E} \nabla_{F} G-\nabla_{F} \nabla_{E} G-\nabla_{[E, F]} G,
$$

for any $E, F, G \in T_{x} \overline{\mathbf{M}}, x \in \overline{\mathbf{M}}$.

1. The connection $\nabla$ is said to be symmetric if its torsion tensor $\mathcal{T}$ vanishes, otherwise it is non-symmetric.

2. The connection $\nabla$ is said to be metric connection if there is a Riemannian metric $g$ in $\overline{\mathbf{M}}$ such that $\nabla g=0$, otherwise it is non-metric.

3. A linear connection $\nabla$ is said to be semi-symmetric connection if its torsion tensor $\mathcal{T}$ is of the form:

$$
\mathcal{T}(E, F)=u(F) E-u(E) F
$$

where $u$ is a 1 -form.

Remark 1. It is well known that a linear connection is symmetric and metric if it is the LeviCivita connection.

The Casorati curvature $\mathbf{C}$ of an $n$-dimensional submanifold $\mathbf{M}$ of a Riemannian manifold $\overline{\mathbf{M}}$ is an extrinsic invariant defined as the normalized square of the length of the second fundamental form $h$ of the submanifold M. In the spirit of $\delta$-invariants, Decu et al. [6] introduced the normalized $\delta^{\prime}$-Casorati curvatures $\delta_{\mathbf{C}}^{\prime}(n-1)$ and $\hat{\delta}_{\mathbf{C}}^{\prime}(n-1)$ in 2007 by

$$
\left[\delta_{\mathbf{C}}^{\prime}(n-1)\right]_{x}=\frac{1}{2} \mathbf{C}_{x}+\frac{n+1}{2 n} \inf \left\{\mathbf{C}(L) \mid \mathrm{L} \text { a hyperplane of } T_{x} \mathbf{M}\right\},
$$

and

$$
\left[\hat{\delta}_{\mathbf{C}}^{\prime}(n-1)\right]_{x}=2 \mathbf{C}_{x}-\frac{2 n-1}{2 n} \sup \left\{\mathbf{C}(L) \mid \mathrm{L} \text { a hyperplane of } T_{x} \mathbf{M}\right\}
$$

where $x \in \mathbf{M}$, and proved some inequalities involving these $\delta^{\prime}$-invariants for submanifolds in real space forms endowed with semi-symmetric metric connections. Since then many researchers obtained such inequalities for different submanifolds in different ambient spaces (for more details, see [5] and references therein). In this article, we will establish the inequalities in terms of $\delta_{\mathbf{C}}^{\prime}(n-1)$ for submanifolds of $\delta$-Lorentzian trans-Sasakian manifold with a semi-symmetric metric connection. 


\section{Preliminaries}

Let $\overline{\mathbf{M}}$ be a $\delta$-almost contact metric manifold equipped with $\delta$-almost contact metric structure $(\phi, \xi, \eta, g, \delta)$ consisting of a $(1,1)$ tensor field $\phi$, a vector field $\xi$, a 1 -form $\eta$ and an indefinite metric $g$ such that

$$
\begin{gathered}
\phi^{2}=E+\eta(E) \xi, \quad \eta \circ \phi=0, \quad \phi \xi=0, \\
\eta(\xi)=-1, \\
g(\xi, \xi)=-\delta \\
\eta(E)=\delta g(E, \xi)
\end{gathered}
$$

and

$$
g(\phi E, \phi F)=g(E, F)+\delta \eta(E) \eta(F),
$$

for any $E, F \in T_{x} \overline{\mathbf{M}}, x \in \overline{\mathbf{M}}$, where $\delta$ is such that $\delta^{2}=1$ so that $\delta= \pm 1$. The above structure $(\phi, \xi, \eta, g, \delta)$ on $\overline{\mathbf{M}}$ is called the $\delta$ Lorentzian structure on $\overline{\mathbf{M}}$. If $\delta=1$ and this is usual Lorentzian structure [1] on $\overline{\mathbf{M}}$, the vector field $\xi$ is the time like [17], that is $\overline{\mathbf{M}}$ contains a time like vector field. If $\delta=-1$, then $\overline{\mathbf{M}}$ is NOT a Lorentzian manifold but a Riemannian one.

Tanno gave the complete classification of connected almost contact metric manifold in [28]. For such a manifold, the sectional curvature of the plane section containing $\xi$ is constant, say $c$. Then Tanno proved that they can be divided into three classes: homogeneous normal contact Riemannian manifolds with $c>0$ and other two classes can be observed in [28].

On the other hand, in the classification of almost Hermitian manifolds, there develop a class $W_{4}$ of Hermitian manifolds which is merely related to the conformal Kaehler manifolds [9]. The class $C_{6} \oplus C_{5}$ consist the structure namely trans-Sasakian of type $(\alpha, \beta)$ (see [16]) and this class completely explains the characteristics of trans-Sasakian structures.

Now, suppose an almost contact metric structure [4] on $\overline{\mathbf{M}}$ and a product manifold $\mathcal{M}=$ $\overline{\mathbf{M}} \times \mathbb{R}$ with the product metric $\mathbb{G}$ on $\mathcal{M}$. Then $\overline{\mathbf{M}}$ is called a trans-Sasakian [21] if $(\mathcal{M}, J, \mathbb{G})$ belongs to the class $W_{4}$, where $J$ is the almost complex structure on $\mathcal{M}$ defined by

$$
J\left(E, \psi \frac{d}{d t}\right)=\left(\phi(E)-\psi \xi, \eta(E) \frac{d}{d t}\right),
$$

for any $E \in T_{x} \overline{\mathbf{M}}, x \in \overline{\mathbf{M}}$ and smooth functions $\psi$ on $\mathcal{M}$.

This may be expressed by the following condition:

$$
\left(\nabla_{E} \phi\right) F=\alpha(g(E, F) \xi-\eta(F) E)+\beta(g(\phi E, F) \xi-\eta(F) \phi E),
$$

for any $E, F \in T_{x} \overline{\mathbf{M}}, \nabla$ denotes the Levi-Civita connection with respect to $g, \alpha$ and $\beta$ are smooth functions on $\overline{\mathbf{M}}$.

With the above literature, we recall the $\delta$-Lorentzian trans-Sasakian manifolds [1] as follows. 
Definition 1. A $\delta$-Lorentzian manifold with structure $(\phi, \xi, \eta, g, \delta)$ is said to be $\delta$-Lorentzian trans-Sasakian manifold of type $(\alpha, \beta)$ if it satisfies the condition

$$
\left(\nabla_{E} \phi\right) F=\alpha(g(E, F) \xi-\delta \eta(F) E)+\beta(g(\phi E, F) \xi-\delta \eta(F) \phi E)
$$

for any $E, F \in T_{x} \overline{\mathbf{M}}$.

1. If $\delta=1$, then the $\delta$-Lorentzian trans-Sasakian manifold is the usual Lorentzian transSasakian manifold of type $(\alpha, \beta)[21]$.

2. $\delta$-Lorentzian trans-Sasakian manifold of type $(0,0),(0, \beta)(\alpha, 0)$ are respectively known as Lorentzian cosymplectic manifold, Lorentzian $\beta$-Kenmotsu manifold and Lorentzian $\alpha$ Sasakian manifold.

3. If $\alpha=1, \beta=0$ and $\alpha=0, \beta=1$, the $\delta$-Lorentzian trans-Sasakian manifold become $\delta$-Lorentzian Sasakian and $\delta$-Lorentzian Kenmotsu manifolds respectively.

Also, we have

$$
\nabla_{E} \xi=\delta\{-\alpha \phi(E)-\beta(E+\eta(E) \xi\}
$$

and

$$
\left(\nabla_{E} \eta\right) F=\alpha g(\phi E, F)+\beta[g(E, F)+\delta \eta(E) \eta(F)] .
$$

In a $\delta$-Lorentzian trans-Sasakian manifold $\overline{\mathbf{M}}$, we have the following relations:

$$
\begin{aligned}
& R(E, F) \xi=\left(\alpha^{2}+\beta^{2}\right)[\eta(F) E-\eta(E) F]+2 \alpha \beta[\eta(F) \phi E-\eta(E) \phi F] \\
& +\delta\left[(F \alpha) \phi E-(E \alpha) \phi F+(Y \beta) \phi^{2} E-(E \beta) \phi^{2} F\right] \text {, } \\
& R(\xi, F) E=\left(\alpha^{2}+\beta^{2}\right)[\delta g(E, F) \xi-\eta(E) F] \\
& +\delta(E \alpha) \phi F+\delta g(\phi E, F)(\operatorname{grad} \alpha) \\
& +\delta(E \beta)(F+\eta(F) \xi)-\delta g(\phi F, \phi E))(\operatorname{grad} \beta) \\
& +2 \alpha \beta[\delta g(\phi E, F) \xi+\eta(E) \phi F] \text {, } \\
& \eta(R(E, F) G)=\delta\left(\alpha^{2}+\beta^{2}\right)[\eta(E) g(F, G)-\eta(F) g(E, G) \\
& +2 \delta \alpha \beta[-\eta(E) g(\phi F, G)+\eta(F) g(\phi E, G)] \\
& -[(F \alpha) g(\phi E, G)+(E \alpha) g(F, \phi G)] \\
& \left.-(F \beta) g\left(\phi^{2} E, G\right)+(E \beta) g\left(\phi^{2} F, G\right)\right] \text {, } \\
& \operatorname{Ric}(E, \xi)=\left[(m-1)\left(\alpha^{2}+\beta^{2}\right)-(\xi \beta)\right] \eta(E) \\
& +\delta((\phi E) \alpha)+(m-2) \delta(E \beta) \text {, } \\
& \operatorname{Ric}(\xi, \xi)=(m-1)\left(\alpha^{2}+\beta^{2}\right)-\delta(m-1)(\xi \beta),
\end{aligned}
$$


where $R$ is Riemannian curvature tensor and Ric is the Ricci curvature tensor with respect to $\nabla$.

Furthermore in an $\delta$-Lorentzian trans-Sasakian manifold, we have

$$
\delta \phi(\operatorname{grad} \alpha)=\delta(m-2)(\operatorname{grad} \beta),
$$

and

$$
2 \alpha \beta-\delta(\xi \alpha)=0 .
$$

The $\xi$-sectional curvature $K_{\xi}$ of $\overline{\mathbf{M}}$ is the sectional curvature of the plane spanned by $\xi$ and a unit vector field $E$. From (2.11), we have

$$
K_{\xi}=g(R(\xi, E), \xi, E)=\left(\alpha^{2}+\beta^{2}\right)-\delta(\xi \beta) .
$$

It follows from (2.17) that $\xi$-sectional curvature does not depend on $E$. From (2.11), we have

$$
\begin{aligned}
g(R(\xi, F) G, \xi)= & {\left[\left(\alpha^{2}+\beta^{2}\right)-\delta(\xi \beta)\right] g(F, G) } \\
& +\left[(\xi \beta)-\delta\left(\alpha^{2}+\beta^{2}\right)\right] \eta(F) \eta(G) \\
& +[2 \alpha \beta+\delta(\delta \alpha)] g(\phi F, G) .
\end{aligned}
$$

Semi-symmetric metric connections $(S S M)$ play an important role in the study of Riemannian manifolds. There are various physical problems involving the $S S M$ connection. For example, during the mathematical congress in Moscow in 1934, one evening mathematicians invented the Moscow displacement. The streets of Moscow are approximately straight lines through the Kremlin and concentric circles around it. If a person walks in the street always facing the Kremlin, then this displacement is semi-symmetric and metric [7].

An affine connection $\bar{\nabla}$ on Riemannian manifold $\overline{\mathbf{M}}$ is called semi-symmetric connection [7], if torsion tensor $\overline{\mathcal{T}}$ satisfies

$$
\overline{\mathcal{T}}(E, F)=\bar{\nabla}_{E} F-\bar{\nabla}_{E} F-[E, F]
$$

and

$$
\overline{\mathcal{T}}(E, F)=\eta(E) F-\eta(F) E .
$$

Moreover, a semi-symmetric connection is called semi-symmetric metric connection $(S S M)$ if

$$
(\bar{\nabla} g)(E, F)=0 .
$$

If $\nabla$ is Levi-Civita connection and $\bar{\nabla}$ is the semi-symmetric metric connection with nonvanishing torsion tensor $\mathcal{T}$ in $\overline{\mathbf{M}}$, then we have

$$
\begin{gathered}
\mathcal{T}(E, F)=\eta(F) E-\eta(E) F, \\
\bar{\nabla}_{E} F-\nabla_{E} F=\frac{1}{2}\left[\mathcal{T}(E, F)+\mathcal{T}^{\prime}(E, F)+\mathcal{T}^{\prime}(E, F)\right],
\end{gathered}
$$

where

$$
g(\mathcal{T}(G, E), F)=g\left(\mathcal{T}^{\prime}(E, F), G\right) .
$$

By using (2.4), (2.22) and (2.23), we get

$$
g\left(\mathcal{T}^{\prime}(E, F), G\right)=g(\eta(E) G-\eta(G) E, F),
$$




$$
\begin{gathered}
g\left(\mathcal{T}^{\prime}(E, F), G\right)=\eta(E) g(G, F)-\delta g(E, F) g(\xi, G), \\
\mathcal{T}^{\prime}(E, F)=\eta(E) F-\delta g(E, F) \xi \\
\mathcal{T}^{\prime}(F, E)=\eta(F) E-\delta g(E, F) \xi
\end{gathered}
$$

From $(2.21),(2.22),(2.24)$ and $(2.25)$, we get

$$
\bar{\nabla}_{E} F=\nabla_{E} F+\eta(F) E-\delta g(E, F) \xi
$$

Let $\overline{\mathbf{M}}$ be an $m$-dimensional $\delta$-Lorentzian trans-Sasakian manifold and $\nabla$ be the metric connection on $\overline{\mathbf{M}}$. The relation between the semi-symmetric metric connection $\bar{\nabla}$ and the metric connection $\nabla$ on $\overline{\mathbf{M}}$ is given by $(2.27)$.

\section{Estimation of curvatures on $\delta$-Lorentzian trans-Sasakian manifold with a $S S M$ connection}

Let $\overline{\mathbf{M}}$ be an $m$-dimensional $\delta$-Lorentzian trans-Sasakian manifold. The curvature tensor $\bar{R}$ of $\overline{\mathbf{M}}$ with respect to the $S S M$ connection $\bar{\nabla}$ is defined by

$$
\bar{R}(E, F) G=\bar{\nabla}_{E} \bar{\nabla}_{F} G-\bar{\nabla}_{F} \bar{\nabla}_{E} G-\bar{\nabla}_{[E, F]} G .
$$

By using (2.1), (2.4), (2.27) and (3.1), we get

$$
\begin{aligned}
& \bar{R}(E, F) G=R(E, F) G+(\delta)[g(E, G) F-g(F, G) E] \\
&+(\beta+\delta)[g(F, G) \eta(E)-g(E, G) \eta(F)] \xi \\
&-(\beta \delta-1)[\eta(F) E-\eta(E) F] \eta(G), \\
&+\alpha[g(\phi E, G) F-g(\phi F, G) \phi E-g(E, G) \phi F+g(F, G) \phi E] .
\end{aligned}
$$

Lemma 3.1. Let $\overline{\mathbf{M}}$ be an m-dimensional $\delta$-Lorentzian trans-Sasakian manifold with a SSM connection, then

$$
\begin{gathered}
\left(\bar{\nabla}_{E} \phi\right)(F)=\alpha g(\phi E, F) \xi-\delta \eta(F) E+\beta(g(\phi E, F) \xi-(\delta \beta+\delta) \eta(F) \phi E \\
\bar{\nabla}_{E} \xi=-(1+\delta \beta) E-(1+\delta \beta) \eta(E) \xi-\delta \alpha \phi E \\
\left(\bar{\nabla}_{E} \eta\right) F=\alpha g(\phi E, F) \xi+(\beta+\delta) g(E, F)-(1+\beta \delta) \eta(E) \eta(F) .
\end{gathered}
$$


Proof. By the covariant differentiation of $\phi F$ with respect to $E$, we have

$$
\bar{\nabla}_{E} \phi F=\left(\bar{\nabla}_{E} \phi\right)+\phi\left(\bar{\nabla}_{E} F\right) .
$$

By using (2.1) and (2.26), we have

$$
\left(\bar{\nabla}_{E} \phi\right) F=\left(\nabla_{E} \phi\right) F-\eta(F) \phi E .
$$

In view of (2.8), the last equation gives

$$
\left(\bar{\nabla}_{E} \phi\right)(F)=\alpha(g(\phi E, F) \xi-\delta \eta(F) E+\beta(g(\phi E, F) \xi-(\delta \beta+\delta) \eta(F) \phi E .
$$

To prove (3.4), we replace $F=\xi$ in $(2.26)$ and we have

$$
\bar{\nabla}_{E} \xi=\nabla_{E} \xi+\eta(\xi) E-\delta g(E, \xi) \xi .
$$

By using (2.2), (2.4) and (2.9), the above equation gives

$$
\bar{\nabla}_{E} \xi=-(1+\delta \beta) E-(1+\delta \beta) \eta(E) \xi-\delta \alpha \phi E .
$$
we have

In order to prove (3.5), we differentiate $\eta(F)$ covariantly with respect to $E$ and using (2.26),

$$
\bar{\nabla}_{E} \eta(Y)=\left(\nabla_{E} \eta\right) F+g(E, F)-\eta(E) \eta(F) .
$$

Using (2.10) in above equation, we get

$$
\left(\bar{\nabla}_{E} \eta\right) F=\alpha g(\phi E, F) \xi+(\beta+\delta) g(E, F)-(1+\beta \delta) \eta(E) \eta(F) .
$$

Lemma 3.2. Let $\overline{\mathbf{M}}$ be an $m$-dimensional $\delta$-Lorentzian trans-Sasakian manifold with a SSM connection, then

$$
\begin{gathered}
\bar{R}(E, F) \xi=\left(\alpha^{2}+\beta^{2}-\delta \beta\right)[\eta(E) F-\eta(F) E] . \\
+(2 \alpha \beta+\delta \alpha)[\eta(F) \phi E-\eta(E) \phi F] \\
+\delta\left[(F \alpha) \phi E-(E \alpha) \phi F-(E \beta) \phi^{2} F+(F \beta) \phi^{2} E\right] .
\end{gathered}
$$

Proof. By replacing $G=\xi$ in (3.2), we have

$$
\begin{gathered}
\bar{R}(E, F) \xi=R(E, F) \xi+(\delta)[g(E, \xi) F-g(F, \xi) E] \\
+(\beta+\delta)[g(F, \xi) \eta(E)-g(E, \xi) \eta(F)] \xi \\
-(\beta \delta-1)[\eta(F) E-\eta(E) F] \eta(\xi) \\
+\alpha[g(\phi E, \xi) F-g(\phi F, \xi) \phi E-g(E, \xi) \phi F+g(F, \xi) \phi E]
\end{gathered}
$$

In view of (2.2), (2.4) and (2.10), the above equation reduces to

$$
\bar{R}(E, F) \xi=\left(\alpha^{2}+\beta^{2}-\delta \beta\right)[\eta(E) F-\eta(F) E]
$$




$$
\begin{gathered}
+(2 \alpha \beta+\delta \alpha)[\eta(F) \phi E-\eta(E) \phi F] \\
+\delta\left[(F \alpha) \phi E-(E \alpha) \phi F-(E \beta) \phi^{2} F+(F \beta) \phi^{2} E\right] .
\end{gathered}
$$

Remark 2. Replace $F=\xi$ and using (3.2), (2.11), (2.2) and (2.4), we find

$$
\begin{gathered}
\bar{R}(E, \xi) \xi=\left(\alpha^{2}+\beta^{2}-\delta \beta\right)[-E-\eta(E) F] \\
+(2 \alpha \beta+\delta \alpha+\delta(\xi \alpha))\left[\phi E+\delta(\xi \beta) \phi^{2} F\right] .
\end{gathered}
$$

Remark 3. Now, again replace $E=\xi$ in (3.6), using (2.1), (2.2) and (2.4), we find

$$
\begin{gathered}
\bar{R}(\xi, F) \xi=\left(\alpha^{2}+\beta^{2}-\delta \beta\right)[-\eta(F) \xi-F] \\
-(2 \alpha \beta+\delta \alpha+\delta(\xi \alpha))\left[\phi F-\delta(\xi \beta) \phi^{2} F\right] .
\end{gathered}
$$

Remark 4. Replace $F=E$ in (3.8), we get

$$
\begin{gathered}
\bar{R}(\xi, E) \xi=-\left(\alpha^{2}+\beta^{2}-\delta \beta\right)[-E-\eta(E) \xi] . \\
-(2 \alpha \beta+\delta \alpha+\delta(\xi \alpha))\left[\phi E-\delta(\xi \beta) \phi^{2} E\right] .
\end{gathered}
$$

From (3.8) and (3.9), we obtain

$$
\bar{R}(E, \xi) \xi=-\bar{R}(\xi, E) \xi
$$

Now, using contraction on $E$ in (3.2), we get

$$
\begin{aligned}
\overline{\operatorname{Ric}}(F, G)= & \operatorname{Ric}(E, G)-[(\delta)(m-2)+\beta] g(F, G) \\
& -(\beta \delta-1)(m-2) \eta(Z) \eta(Y)-\alpha(m-2) g(\phi F, G),
\end{aligned}
$$

where Ric and Ric are the Ricci tensors of the connections $\bar{\nabla}$ and $\nabla$, respectively on $\overline{\mathbf{M}}$.

Putting $F=G=e_{i}$ and taking summation over $i, 1 \leq i \leq m-1$ in (3.12), using (2.15) and also the relations $\tau=\operatorname{Ric}\left(e_{i}, e_{i}\right)=\sum_{i=1}^{m} \delta_{i} R\left(e_{i}, e_{i}, e_{i}, e_{i}\right)$, we get

$$
\bar{\tau}=\tau-(m-1)[(\delta)(m-2)+2 \beta],
$$

where $\bar{\tau}$ and $\tau$ are the scalar curvatures of the connections $\bar{\nabla}$ and $\nabla$, respectively on $\overline{\mathbf{M}}$.

Now, we have the following lemmas:

Lemma 3.3. Let $\overline{\mathbf{M}}$ be an $m$-dimensional $\delta$-Lorentzian trans-Sasakian manifold with the SSM connection, then the scalar curvature is constant. 


\section{Examples of $\delta$-Lorentzian trans-Sasakiam manifolds}

In [13], we found a non trivial example of $\delta$-Lorentzian trans-Sasakian manifold with a $S S M$ connection.

Example 1. The three dimensional manifold $\overline{\mathbf{M}}=\left\{(x, y, z) \in \mathbb{R}^{3} \mid z \neq 0\right\}$, where $(x, y, z)$ are the Cartesian coordinates in $\mathbb{R}^{3}$, is a $\delta$-Lorentzian trans-Sasakian of type $(0,1)$ manifold. Here the Riemannian metric $g$ is given by

$$
g\left(v_{1}, v_{3}\right)=g\left(v_{2}, v_{3}\right)=g\left(v_{2}, v_{2}\right)=0, \quad g\left(v_{1}, v_{1}\right)=g\left(v_{2}, v_{2}\right)=g\left(v_{3}, v_{3}\right)=\delta,
$$

where $\delta= \pm 1$ and $v_{1}, v_{2}, v_{3}$ are vector fields. And the 1 -form $\eta$ is defined by $\eta(G)=\delta g\left(G, v_{3}\right)$, for any $G \in T_{x} \overline{\mathbf{M}}$. The $(1,1)$ tensor field $\phi$ is defined by $\phi\left(v_{1}\right)=-v_{2}, \phi\left(v_{2}\right)=e_{1}, \phi\left(v_{3}\right)=0$.

From Koszul's formula, we have

$$
\begin{aligned}
& \nabla_{v_{1}} v_{3}=\delta v_{1}, \quad \nabla_{v_{2}} v_{3}=\delta v_{2}, \quad \nabla_{v_{3}} v_{3}=0, \\
& \nabla_{v_{1}} v_{2}=0, \quad \nabla_{v_{2}} v_{2}=-\delta v_{3}, \quad \nabla_{v_{3}} v_{2}=0 \text {, } \\
& \nabla_{v_{1}} v_{1}=-\delta v_{3}, \quad \nabla_{v_{2}} v_{1}=0, \quad \nabla_{v_{3}} v_{1}=0 .
\end{aligned}
$$

Also, for any vector field $E$ on $\overline{\mathbf{M}}$, we have

$$
\nabla_{E} \xi=\delta(E+\eta(X) \xi)
$$

for $\xi \in v_{3}, \alpha=0$ and $\beta=1$.

The semi-symmetric metric connection on $\overline{\mathbf{M}}$ is given below:

$$
\begin{gathered}
\bar{\nabla}_{v_{1}} v_{3}=(1+\delta) v_{1}, \quad \bar{\nabla}_{v_{2}} v_{3}=(1+\delta) v_{2}, \quad \bar{\nabla}_{v_{3}} v_{3}=0, \\
\bar{\nabla}_{v_{1}} v_{2}=0, \quad \bar{\nabla}_{v_{2}} v_{2}=-(1+\delta) v_{3}, \quad \bar{\nabla}_{v_{3}} v_{2}=0 \\
\bar{\nabla}_{v_{1}} v_{1}=-(1+\delta) v_{3}, \quad \bar{\nabla}_{v_{2}} v_{1}=0, \quad \bar{\nabla}_{v_{3}} v_{1}=0 .
\end{gathered}
$$

Example 2. We consider the three dimensional manifold $\overline{\mathbf{M}}=\left\{(x, y, z) \in \mathbb{R}^{3} \mid z \neq 0\right\}$, where $(x, y, z)$ are the Cartesian coordinates in $\mathbb{R}^{3}$. Choosing the vector fields

$$
v_{1}=z\left(\frac{\partial}{\partial x}+y \frac{\partial}{\partial z}\right), \quad v_{2}=z \frac{\partial}{\partial y}, \quad v_{3}=\frac{\partial}{\partial z},
$$

which are linearly independent vector fields at each point $x$ of $\overline{\mathbf{M}}$. The Lorentzian metric $g$ is defined by

$$
g\left(v_{1}, v_{3}\right)=g\left(v_{1}, v_{2}\right)=g\left(v_{2}, v_{3}\right)=0, \quad g\left(v_{1}, v_{1}\right)=g\left(v_{2}, v_{2}\right)=1, \quad g\left(v_{3}, v_{3}\right)=-1 \quad(\delta=1) .
$$

For any $U \in T_{x} \overline{\mathbf{M}}$, the 1 -form $\eta$ is given as $\eta(U)=\delta g\left(U, v_{3}\right)$. The $(1,1)$ tensor field $\phi$ is defined by $\phi\left(v_{1}\right)=-v_{2}, \phi\left(v_{2}\right)=-v_{1}, \phi\left(v_{3}\right)=0$. Then, by the linearity property of $\phi$ and $g$, we have

$$
\phi^{2} U=U+\eta(U) v_{3}, \quad \eta\left(v_{3}\right)=-1, \quad g(\phi U, \phi V)=g(U, V)+\delta \eta(U) \eta(V),
$$

for any $U, V \in T_{x} \overline{\mathbf{M}}, x \in \overline{\mathbf{M}}$. Then for a timelike vector field $\xi=v_{3}$, the structure $(\phi, \xi, \eta, g, \delta=1)$ defines a Lorentzian structure on $\overline{\mathbf{M}}$. 
Let $\nabla$ be the Levi-Civita connection with respect to the metric $g$. Then, we have

$$
\left[v_{1}, v_{2}\right]=y v_{2}-z^{2} v_{3}, \quad\left[v_{1}, v_{3}\right]=-\frac{1}{z} v_{1}, \quad\left[v_{2}, v_{3}\right]=-\frac{1}{z} v_{2} .
$$

The Riemannian connection $\nabla$ with respect to the metric $g$ is given by

$$
\begin{aligned}
2 g\left(\nabla_{E} F, G\right)= & E g(F, G)+F g(G, E)-G g(E, F)+g([E, F], G) \\
& -g([F, G], E)+g([G, E], F) .
\end{aligned}
$$

From above equation which is known as Koszul's formula, we have

$$
\begin{gathered}
\nabla_{v_{1}} v_{1}=-\frac{1}{z} v_{3}, \quad \nabla_{v_{1}} v_{3}=-\frac{1}{z} v_{1}+\frac{1}{z^{2}} v_{2}, \quad \nabla_{v_{1}} v_{2}=-\frac{1}{2} z^{2} v_{3}, \\
\nabla_{v_{2}} v_{3}=-\frac{1}{z} v_{1}+\frac{1}{2} z^{2} v_{1}, \quad \nabla_{v_{2}} v_{2}=y v_{3}-\frac{1}{z} v_{3}, \quad \nabla_{v_{2}} v_{1}=\frac{1}{2} z^{2} v_{3}-y v_{2}, \\
\nabla_{v_{3}} v_{3}=0, \quad \nabla_{v_{3}} v_{2}=\frac{1}{2} z^{2} v_{1}, \quad \nabla_{v_{3}} v_{1}=-\frac{1}{2} z^{2} v_{3} .
\end{gathered}
$$

From the above it can be easily observe that $(\phi, \xi, \eta, g, \delta=1)$ is a Lorentzian trans-Sasakian structure on $\overline{\mathbf{M}}$. Therefore $\overline{\mathbf{M}}$ is a Lorentzian trans-Sasakian manifolds with $\alpha=\frac{1}{2} z^{2} \neq 0$ and $\beta=\frac{1}{z} \neq 0$. Hence the manifold $\overline{\mathbf{M}}$ under consideration is an $\delta$-Lorentzian trans-Sasakian of type $(\alpha, \beta)$ manifold of dimension three.

Example 3. We consider the 3-dimensional manifold $\overline{\mathbf{M}}=\left\{(x, y, z) \in \mathbb{R}^{3} \mid z \neq 0\right\}$, where $(x, y, z)$ are the Cartesian coordinates in $\mathbb{R}^{3}$. Choosing the vector fields

$$
v_{1}=\frac{\partial}{\partial x}+\frac{\partial}{\partial y}, \quad v_{2}=-\frac{\partial}{\partial x}+\frac{\partial}{\partial y}, \quad v_{3}=\frac{\partial}{\partial z},
$$

which are linearly independent vector fields at each point $x$ of $\overline{\mathbf{M}}$. The Lorentzian metric $g$ is defined as

$$
g\left(v_{1}, v_{2}\right)=g\left(v_{1}, v_{3}\right)=g\left(v_{3}, v_{2}\right)=0, \quad g\left(v_{1}, v_{1}\right)=g\left(v_{2}, v_{2}\right)=1, \quad g\left(v_{3}, v_{3}\right)=-\delta,
$$

where $\delta= \pm 1$. Then $\delta$-Lorentzian infinite metric $g$ on $\overline{\mathbf{M}}$ is in the following form:

$$
g=(d x)^{2}+(d y)^{2}-\delta(d z)^{2} .
$$

Let $v_{3}=\xi$ be a timelike vector filed and $\eta$ be the 1-form defined by $\eta(U)=\delta g\left(U, v_{3}\right)$, for any $U \in T_{x} \overline{\mathbf{M}}$. The $(1,1)$ tensor field $\phi$ is defined by $\phi\left(v_{1}\right)=-v_{1}, \phi\left(v_{2}\right)=-v_{2}, \phi\left(v_{3}\right)=0$. Then by the linearity property of $\phi$ and $g$, and taking $v_{3}=\xi$, we have

$$
\begin{aligned}
& \phi^{2} U=U+\eta(U) v_{3}, \quad \eta\left(v_{3}\right)=-1, \\
& g(\phi U, \phi V)=g(U, V)+\delta \eta(U) \eta(V)
\end{aligned}
$$

for any vector fields $U, V \in T_{x} \overline{\mathbf{M}}, x \in \overline{\mathbf{M}}$.

Now putting $V=\xi$ in (4.2), we turn up

$$
\eta(U)=\delta g(U, \xi)
$$

Putting $V=U=\xi$ in (4.2) and (4.3) respectively, we get

$$
g(\xi, \xi)=-\delta, \quad \eta(\xi)=-1 .
$$

Since $\overline{\mathbf{M}}$ satisfies all conditions (2.1)-(2.6) of $\delta$-Lorentzian trans-Sasakian manifolds, $\overline{\mathbf{M}}$ is a three dimensional $\delta$-Lorentzian trans-Sasakian manifolds. 
Example 4. We consider the 3-dimensional manifold $\overline{\mathbf{M}}=\left\{(x, y, z) \in \mathbb{R}^{3} \mid z \neq 0\right\}$, where $(x, y, z)$ are the Cartesian coordinates in $\mathbb{R}^{3}$. Choosing the vector fields

$$
v_{1}=\cosh z \frac{\partial}{\partial x}+\sinh z \frac{\partial}{\partial y}, \quad v_{2}=\sinh z \frac{\partial}{\partial x}+\cosh z \frac{\partial}{\partial y}, \quad v_{3}=\frac{\partial}{\partial z},
$$

which are linearly independent vector fields at each point $x$ of $\overline{\mathbf{M}}$.

Let $g$ be the Lorentzian metric define by

$$
g\left(v_{1}, v_{2}\right)=g\left(v_{1}, v_{3}\right)=g\left(v_{3}, v_{2}\right)=0, \quad g\left(v_{1}, v_{1}\right)=g\left(v_{2}, v_{2}\right)=1, g\left(v_{3}, v_{3}\right)=-\delta,
$$

where $\delta= \pm 1$.

Let $v_{3}=\xi$ is a timelike vector filed and $\eta$ be the 1-form defined by $\eta(U)=\delta g\left(U, v_{3}\right)$, for any $U \in T_{x} \overline{\mathbf{M}}$. The $(1,1)$ tensor field $\phi$ is defined by $\phi\left(v_{1}\right)=-v_{1}, \phi\left(v_{2}\right)=-v_{2}, \phi\left(v_{3}\right)=0$. Then by the linearity property of $\phi$ and $g$, and taking $v_{3}=\xi$, we have

$$
\begin{gathered}
\phi^{2} U=U+\eta(U) v_{3}, \quad \eta\left(v_{3}\right)=-1, \\
g(\phi U, \phi V)=g(U, V)+\delta \eta(U) \eta(V),
\end{gathered}
$$

for any vector fields $U, V \in T_{x} \overline{\mathbf{M}}, x \in \overline{\mathbf{M}}$.

Now, putting $V=\xi$ in (4.2), we turn up

$$
\eta(U)=\delta g(U, \xi)
$$

Putting $V=U=\xi$ in (4.5) and (4.6) respectively we get

$$
g(\xi, \xi)=-\delta, \quad \eta(\xi)=-1 .
$$

Since $\overline{\mathbf{M}}$ satisfies all conditions (2.1)-(2.6) of $\delta$-Lorentzian trans-Sasakian manifolds, $\overline{\mathbf{M}}$ is a three dimensional $\delta$-Lorentzian trans-Sasakian manifolds.

Let $\nabla$ be the Levi-Civita connection with respect to the metric $g$. Then, we have

$$
\left[v_{1}, v_{2}\right]=0, \quad\left[v_{3}, v_{1}\right]=v_{2}, \quad\left[v_{2}, v_{3}\right]=-v_{1} .
$$

Using Koszul's formula, we can easily calculate

$$
\begin{array}{ccc}
\nabla_{v_{1}} v_{1}=0, \quad \nabla_{v_{1}} v_{3}=-v_{2}, & \nabla_{v_{1}} v_{2}=-v_{3}, \\
\nabla_{v_{2}} v_{3}=-v_{1}, \quad \nabla_{v_{2}} v_{2}=0, & \nabla_{v_{2}} v_{1}=-v_{3}, \\
\nabla_{v_{3}} v_{3}=0, \quad \nabla_{v_{3}} v_{2}=0, & \nabla_{v_{3}} v_{1}=0 .
\end{array}
$$

From the above it can be easily observe that $(\phi, \xi, \eta, g, \delta=1)$ is a Lorentzian trans-Sasakian structure on $\overline{\mathbf{M}}$ with $\alpha=-1$ and $\beta=0$. Hence the manifold $\overline{\mathbf{M}}$ under consideration is an $\delta$-Lorentzian trans-Sasakian of type $(\alpha, 0)$ manifold of dimension three.

Example 5. We consider the 3-dimensional manifold $\overline{\mathbf{M}}=\left\{(x, y, z) \in \mathbb{R}^{3} \mid z \neq 0\right\}$, where $(x, y, z)$ are the Cartesian coordinates in $\mathbb{R}^{3}$. Choosing the vector fields

$$
v_{1}=e^{z} \frac{\partial}{\partial y}, \quad v_{2}=e^{z}\left(\frac{\partial}{\partial x}+\frac{\partial}{\partial y}\right), \quad v_{3}=\frac{\partial}{\partial z},
$$


which are linearly independent vector fields at each point $x$ of $\overline{\mathbf{M}}$.

Let $g$ be the Lorentzian metric define by

$$
g\left(v_{1}, v_{2}\right)=g\left(v_{1}, v_{3}\right)=g\left(v_{3}, v_{2}\right)=0, \quad g\left(v_{1}, v_{1}\right)=g\left(v_{2}, v_{2}\right)=1, \quad g\left(v_{3}, v_{3}\right)=-\delta,
$$

where $\delta= \pm 1$. Then $\delta$-Lorentzian infinite metric $g$ on $\overline{\mathbf{M}}$ is in the following form:

$$
g=e^{-2 z}\left[2(d x)^{2}+(d y)^{2}-2 d x d y\right]-\delta(d z)^{2}
$$

Let $v_{3}=\xi$ is a timelike vector filed, and $\eta$ be the 1-form defined by $\eta(U)=\delta g\left(U, v_{3}\right)$ for any $U \in T_{x} \overline{\mathbf{M}}$.

Let $\phi$ be the $(1,1)$ tensor field defined by $\phi\left(v_{1}\right)=-v_{1}, \phi\left(v_{2}\right)=-v_{2}, \phi\left(v_{3}\right)=0$. Then by the linearity property of $\phi$ and $g$, and taking $v_{3}=\xi$, we have

$$
\begin{gathered}
\phi^{2} U=U+\eta(U) v_{3}, \quad \eta\left(v_{3}\right)=-1, \\
g(\phi U, \phi V)=g(U, V)+\delta \eta(U) \eta(V),
\end{gathered}
$$

for any vector fields $U, V \in T_{x} \overline{\mathbf{M}}, x \in \overline{\mathbf{M}}$.

Now putting $V=\xi$ in (4.9), we turn up

$$
\eta(U)=\delta g(U, \xi)
$$

Putting $V=U=\xi$ in (4.9) and (4.10) respectively, we get

$$
g(\xi, \xi)=-\delta, \quad \eta(\xi)=-1 .
$$

Since $\overline{\mathbf{M}}$ satisfies all conditions (2.1)-(2.6) of $\delta$-Lorentzian trans-Sasakian manifolds, $\overline{\mathbf{M}}$ is a three dimensional $\delta$-Lorentzian trans-Sasakian manifolds.

Let $\nabla$ be the Levi-Civita connection with respect to the metric $g$. Then, we have

$$
\left[v_{1}, v_{2}\right]=0, \quad\left[v_{2}, v_{3}\right]=-v_{2}, \quad\left[v_{1}, v_{3}\right]=-v_{1} .
$$

The Riemannian connection $\nabla$ with respect to the metric $g$ is given by

$$
\begin{aligned}
2 g\left(\nabla_{E} F, G\right)= & E g(F, G)+F g(G, E)-G g(E, F)+g([E, F], G) \\
& -g([F, G], E)+g([G, E], F) .
\end{aligned}
$$

From above equation which is known as Koszul's formula, we have

$$
\begin{gathered}
\nabla_{v_{1}} v_{1}=-v_{1}, \quad \nabla_{v_{1}} v_{3}=-v_{1}+\frac{1}{z^{2}} v_{2}, \quad \nabla_{v_{1}} v_{2}=0, \\
\nabla_{v_{2}} v_{3}=-v_{2}, \quad \nabla_{v_{2}} v_{2}=-v_{3}-, \quad \nabla_{v_{2}} v_{1}=0 \\
\nabla_{v_{3}} v_{3}=0, \quad \nabla_{v_{3}} v_{2}=0, \quad \nabla_{v_{3}} v_{1}=0 .
\end{gathered}
$$

From the above it can be easily observe that $(\phi, \xi, \eta, g, \delta=1)$ is a Lorentzian trans-Sasakian structure on $\overline{\mathbf{M}}$. Therefore $\overline{\mathbf{M}}$ is a Lorentzian trans-Sasakian manifolds with $\alpha=1$ and $\beta=0$.

Now, we provide a non trivial example of $\delta$-Lorentzian trans-Sasakian manifold with a $S S M$ connection. 
Example 6. We consider the three dimensional manifold $\overline{\mathbf{M}}=\left\{(x, y, z) \in \mathbb{R}^{3} \mid z \neq 0\right\}$, where $(x, y, z)$ are the Cartesian coordinates in $\mathbb{R}^{3}$. Choosing the vector fields

$$
v_{1}=z \frac{\partial}{\partial x}, \quad v_{2}=z \frac{\partial}{\partial y}, \quad v_{3}=-z \frac{\partial}{\partial z},
$$

which are linearly independent at each point $x$ of $\overline{\mathbf{M}}$.

Let $g$ be the Lorentzian metric define by

$$
g\left(v_{1}, v_{3}\right)=g\left(v_{2}, v_{3}\right)=g\left(v_{2}, v_{2}\right)=0, \quad g\left(v_{1}, v_{1}\right)=g\left(v_{2}, v_{2}\right)=1, g\left(v_{3}, v_{3}\right)=-\delta,
$$

where $\delta= \pm 1$. Let $\eta$ be the 1 -form defined by $\eta(G)=\delta g\left(G, v_{3}\right)$, for any $G \in T_{x} \overline{\mathbf{M}}$.

Let $\phi$ be the $(1,1)$ tensor field defined by $\phi\left(v_{1}\right)=-v_{1}, \phi\left(v_{2}\right)=-v_{2}, \phi\left(v_{3}\right)=0$. Then by the linearity property of $\phi$ and $g$, we have

$$
\phi^{2} G=G+\eta(G) v_{3}, \quad \eta\left(v_{3}\right)=-1 \quad \text { and } \quad g(\phi G, \phi H)=g(G, H)+\delta \eta(G) \eta(H),
$$

for any $G, H \in T_{x} \overline{\mathbf{M}}, x \in \overline{\mathbf{M}}$.

Let $\nabla$ be the Levi-Civita connection with respect to the metric $g$. Then, we have

$$
\left[v_{1}, v_{2}\right]=0, \quad\left[v_{1}, v_{3}\right]=\delta v_{1}, \quad\left[v_{2}, v_{3}\right]=\delta v_{2} .
$$

The Riemannian connection $\nabla$ with respect to the metric $g$ is given by

$$
\begin{aligned}
2 g\left(\nabla_{E} F, G\right)= & E g(F, G)+F g(G, E)-G g(E, F)+g([E, F], G) \\
& -g([F, G], E)+g([G, E], F) .
\end{aligned}
$$

From above equation which is known as Koszul's formula, we have

$$
\begin{array}{ll}
\nabla_{v_{1}} v_{3}=\delta v_{1}, \quad \nabla_{v_{2}} v_{3}=\delta v_{2}, & \nabla_{v_{3}} v_{3}=0, \\
\nabla_{v_{1}} v_{2}=0, \quad \nabla_{v_{2}} v_{2}=-\delta v_{3}, & \nabla_{v_{3}} v_{2}=0, \\
\nabla_{v_{1}} v_{1}=-\delta v_{3}, \quad \nabla_{v_{2}} v_{1}=0, & \nabla_{v_{3}} v_{1}=0 .
\end{array}
$$

Using the above relations, for any vector field $E$ on $\overline{\mathbf{M}}$, we have

$$
\nabla_{E} \xi=\delta(E+\eta(X) \xi)
$$

for $\xi \in v_{3}, \alpha=0$ and $\beta=1$. Hence the manifold $\overline{\mathbf{M}}$ under consideration is an $\delta$-Lorentzian trans-Sasakian of type $(0,1)$ manifold of dimension three.

Now, we consider this structure for semi-symmetric metric connection, we obtain

$$
\begin{gathered}
\bar{\nabla}_{v_{1}} v_{3}=(1+\delta) v_{1}, \quad \bar{\nabla}_{v_{2}} v_{3}=(1+\delta) v_{2}, \quad \bar{\nabla}_{v_{3}} v_{3}=0 \\
\bar{\nabla}_{v_{1}} v_{2}=0, \quad \bar{\nabla}_{v_{2}} v_{2}=-(1+\delta) v_{3}, \quad \bar{\nabla}_{v_{3}} v_{2}=0 \\
\bar{\nabla}_{v_{1}} v_{1}=-(1+\delta) v_{3}, \quad \bar{\nabla}_{v_{2}} v_{1}=0, \quad \bar{\nabla}_{v_{3}} v_{1}=0 .
\end{gathered}
$$


Then the Riemannian curvature, Ricci curvature, and scalar curvature tensors with respect to $S S M$ connection $\bar{\nabla}$ are given by

$$
\begin{gathered}
\bar{R}\left(v_{1}, v_{2}\right) v_{2}=-(1+\delta)^{2} v_{1}, \quad \bar{R}\left(v_{1}, v_{3}\right) v_{3}=-\delta(1+\delta) v_{2}, \quad \bar{R}\left(v_{2}, v_{1}\right) v_{1}=-(1+\delta)^{2} v_{2}, \\
\bar{R}\left(v_{2}, v_{3}\right) v_{3}=-\delta(1+\delta) v_{2}, \quad \bar{R}\left(v_{3}, v_{1}\right) v_{1}=\delta(1+\delta) v_{3}, \quad \bar{R}\left(v_{3}, v_{2}\right) v_{2}=-\delta(1+\delta) v_{3}, \\
\overline{R i c}\left(v_{1}, v_{1}\right)=\overline{R i c}\left(v_{2}, v_{2}\right)=-(1+\delta)(1+2 \delta), \quad \overline{R i c}\left(v_{3}, v_{3},\right)=2 \delta(1+\delta)
\end{gathered}
$$

and $\bar{\tau}=-2(1+\delta)^{2}$.

\section{Casorati curvatures}

Let $\mathbf{M}$ be an $n$-dimensional submanifold of an $m$-dimensional $\delta$-Lorentzian trans-Sasakian manifold $\overline{\mathbf{M}}$ with a $S S M$ connection and induced metric $g$. We represent the induced connections on the tangent bundle $T \mathbf{M}$ and $T \mathbf{M}^{\perp}$ of $\mathbf{M}$ by $\nabla^{\mathbf{M}}$ and $\nabla^{\mathbf{M}^{\perp}}$, respectively and denote by $h$ the second fundamental form of $\mathbf{M}$. For any $E, F \in T_{x} \mathbf{M}$, and $N \in T_{x} \mathbf{M}^{\perp}, x \in \mathbf{M}$, we recall the Gauss and Weingarten formulas by

$$
\nabla_{E} F=\nabla_{E}^{\mathrm{M}} F+h(E, F)
$$

and

$$
\nabla_{E}^{\perp} N=-A_{N} E+\nabla_{E}^{\mathrm{M}^{\perp}} N
$$

where $A_{N}$ is used for notation of the shape operator of $\mathbf{M}$ with respect to $N$. The following equation is well-known:

$$
g\left(A_{N} E, F\right)=g(h(E, F), N) .
$$

We also recall the equation of Gauss by

$$
\begin{aligned}
R(E, F, G, H)= & R^{\mathbf{M}}(E, F, G, H)-g(h(E, H), h(F, G)) \\
& +g(h(E, G), h(F, H)),
\end{aligned}
$$

for any $E, F, G, H \in T_{x} \mathbf{M}, x \in \mathbf{M}$. Here $R^{\mathbf{M}}$ is the Riemannian curvature tensor with respect to $\nabla^{\mathbf{M}}$.

For a surface in $\mathbb{E}^{3}$ the Casorati curvature is defined as the normalized sum of the squared principal curvatures. This curvature was preferred by Casorati over the traditional Gauss curvature because the Casorati curvature vanishes if and only if both principal curvatures are zero at the same time and thus corresponds better with the common intuition of curvature.

In [15], Lee, et al. obtained Optimal inequalities for the Casorati curvatures of submanifolds of real space forms endowed with semi-symmetric metric connections. With the help of this reference, we find such relations for an $n$-dimensional submanifold $\mathbf{M}$ of an $m$-dimensional $\delta$-Lorentzian trans-Sasakian manifold $\overline{\mathbf{M}}$ with a $S S M$ connection. Therefore, we choose an orthonormal tangent frame $\left\{e_{1}, \ldots, e_{n}\right\}$ and an orthonormal normal frame $\left\{e_{n+1}, \ldots, e_{m}\right\}$ of $\mathbf{M}$ in an $\delta$-Lorentzian trans-Sasakian manifold $\overline{\mathbf{M}}$. Then the scalar curvature $\tau$ at $x \in \mathbf{M}$ is defined by

$$
\tau(x)=\sum_{i<j} K\left(e_{i} \wedge e_{j}\right)
$$


where $K\left(e_{i} \wedge e_{j}\right), 1 \leq i<j \leq n$, denotes the sectional curvature of $\mathbf{M}$ associated with a plane section spanned by $e_{i}$ and $e_{j}$.

The normalized scalar curvature $\rho$ of $\mathbf{M}$ is defined as

$$
\rho=\frac{2 \tau}{n(n-1)} \text {. }
$$

Let $L$ be a subspace of $T_{x} \mathbf{M}, x \in \mathbf{M}$ of dimension $p \geq 2$ and $\left\{e_{1}, \ldots, e_{n}\right\}$ an orthonormal basis of $L$. The scalar curvature $\tau(L)$ of the $p$-plane section $L$ is given by

$$
\tau(L)=\sum_{1 \leq r<s \leq p} K\left(e_{r} \wedge e_{s}\right) .
$$

The squared norm of $h$ over the dimension $n$ is called the Casorati curvature $\mathbf{C}$ of $n$ dimensional submanifold $\mathbf{M}$ in $\overline{\mathbf{M}}$ of dimension $m$, that is,

$$
n \mathbf{C}=\sum_{a=n+1}^{m}\left(\sum_{i, j=1}^{n}\left(h_{i j}^{a}\right)^{2}\right),
$$

whereby $h_{i j}^{a}=g\left(h\left(e_{i}, e_{j}\right), e_{a}\right)$ are the components of the second fundamental form with respect to given orthonormal bases. And the Casorati curvature $\mathbf{C}(L)$ of the subspace $L$ is defined as

$$
p \mathbf{C}(L)=\sum_{a=n+1}^{m}\left(\sum_{i, j=1}^{p}\left(h_{i j}^{a}\right)^{2}\right) \text {. }
$$

Also, the squared mean curvature of the submanifold $\mathbf{M}$ in $\overline{\mathbf{M}}$ is given by

$$
n^{2}\|\mathbf{H}\|^{2}=\sum_{a=n+1}^{m}\left(\sum_{i=1}^{n} h_{i i}^{a}\right)^{2} .
$$

\section{Upper bounds for $\delta^{\prime}$-Casorati curvature}

From the Gauss equation, the following relation between the scalar curvature, the squared mean curvature and the Casorati curvature hold:

$$
\begin{aligned}
2 \tau= & n(n-1)\left(\alpha^{2}+\beta^{2}-\delta \xi \beta\right)-(n-1)[(\delta)(n-2)+2 \beta] \\
& +n^{2}\|\mathbf{H}\|^{2}-n \mathbf{C} .
\end{aligned}
$$

Next, we define the following function $\mathbf{F}$ as a quadratic polynomial in terms of the components of the second fundamental form:

$$
\begin{aligned}
\mathbf{F}= & \frac{1}{2}(n-1)[n \mathbf{C}+(n+1) \mathbf{C}(L)]-2 \tau+n(n-1)\left(\alpha^{2}+\beta^{2}-\delta \xi \beta\right) \\
& -(n-1)[(\delta)(n-2)+2 \beta] .
\end{aligned}
$$
deduce

By saying that $L$ is spanned by $\left\{e_{1}, \ldots, e_{n-1}\right\}$ (without loss of generality), one can easily

$$
\mathbf{F}=\frac{1}{2}(n-1) \sum_{a=n+1}^{m}\left[\sum_{i, j=1}^{n}\left(h_{i j}^{a}\right)^{2}\right]+\frac{1}{2}(n+1) \sum_{a=n+1}^{m}\left[\sum_{i, j=1}^{n-1}\left(h_{i j}^{a}\right)^{2}\right]-2 \tau
$$




$$
+n(n-1)\left(\alpha^{2}+\beta^{2}-\delta \xi \beta\right)-(n-1)[(\delta)(n-2)+2 \beta] .
$$

By substituting equation (6.1) in the last equation, we have

$$
\begin{aligned}
\mathbf{F}= & \frac{1}{2}(n-1) \sum_{a=n+1}^{m}\left[\sum_{i, j=1}^{n}\left(h_{i j}^{a}\right)^{2}\right]+\frac{1}{2}(n+1) \sum_{a=n+1}^{m}\left[\sum_{i, j=1}^{n-1}\left(h_{i j}^{a}\right)^{2}\right] \\
& +n^{2}\|\mathbf{H}\| \|^{2}-n \mathbf{C} .
\end{aligned}
$$

The simple modification in (6.2) gives us the following:

$$
\begin{aligned}
\mathbf{F}= & \frac{1}{2}(n+1)\left\{\sum_{a=n+1}^{m}\left[\sum_{i, j=1}^{n}\left(h_{i j}^{a}\right)^{2}\right]+\sum_{a=n+1}^{m}\left[\sum_{i, j=1}^{n-1}\left(h_{i j}^{a}\right)^{2}\right]\right\} \\
& -\sum_{a=n+1}^{m}\left(\sum_{i=1}^{n} h_{i i}^{a}\right)^{2} .
\end{aligned}
$$

We rewrite the equation (6.3) as follows:

$$
\begin{aligned}
\mathbf{F}= & \sum_{a=n+1}^{m} \sum_{i=1}^{n-1}\left[n\left(h_{i i}^{a}\right)^{2}+(n+1)\left(h_{i n}^{a}\right)^{2}\right] \\
& +\sum_{a=n+1}^{m}\left[2(n+1) \sum_{1 \leq i<j \leq n-1}\left(h_{i j}^{a}\right)^{2}\right. \\
& \left.-2 \sum_{1 \leq i<j \leq n} h_{i i}^{a} h_{j j}^{a}+\frac{1}{2}(n-1)\left(h_{n n}^{a}\right)^{2}\right] .
\end{aligned}
$$

The critical points $h^{c}=\left(h_{11}^{n+1}, h_{12}^{n+1}, \ldots, h_{n n}^{n+1}, \ldots, h_{11}^{m}, h_{12}^{m}, \ldots, h_{n n}^{m}\right)$ of $\mathbf{F}$ are the solutions of the following partial derivatives of $\mathbf{F}$ with respect to $h_{11}^{a}, \ldots, h_{n n}^{a}, a \in\{n+1, \ldots, m\}$ :

$$
\begin{aligned}
\frac{\partial \mathbf{F}}{\partial h_{i i}^{a}} & =2(n+1) h_{i i}^{a}-2 \sum_{k=1}^{n} h_{k k}^{a}=0, \\
\frac{\partial \mathbf{F}}{\partial h_{n n}^{a}} & =(n-1) h_{n n}^{a}-2 \sum_{k=1}^{n-1} h_{k k}^{a}=0 \\
\frac{\partial \mathbf{F}}{\partial h_{i j}^{a}} & =4(n+1) h_{i j}^{a}=0 \\
\frac{\partial \mathbf{F}}{\partial h_{i n}^{a}} & =2(n+1) h_{i n}^{a}=0
\end{aligned}
$$

where $i, j \in\{1,2, \ldots, n-1\}$. Thus, every solution $h^{c}$ has $h_{i j}^{a}=0$ for $i \neq j$, and the determinant which corresponds to the equations (6.4) and (6.5) is zero (there exist solutions for non-totally geodesic submanifolds). Moreover, the Hessian matrix $\mathbf{H}$ of $\mathbf{F}$ is given by

$$
\mathbf{H}(\mathbf{F})=\left(\begin{array}{ccc}
\mathbf{H}_{\mathbf{1}} & 0 & 0 \\
0 & \mathbf{H}_{\mathbf{2}} & 0 \\
0 & 0 & \mathbf{H}_{\mathbf{3}}
\end{array}\right)
$$


where

$$
\mathbf{H}_{\mathbf{1}}=\left(\begin{array}{ccccc}
2 n & -2 & \ldots & -2 & -2 \\
-2 & 2 n & \ldots & -2 & -2 \\
\vdots & \vdots & \ddots & \vdots & \vdots \\
-2 & -2 & \ldots & 2 n & -2 \\
-2 & -2 & \ldots & -2 & n-1
\end{array}\right)
$$

0 are the null matrices and $\mathbf{H}_{\mathbf{2}}$ and $\mathbf{H}_{\mathbf{3}}$ are the diagonal matrices of the respective dimensions.

$$
\begin{aligned}
& \mathbf{H}_{\mathbf{2}}=\operatorname{diag}(4(n+1), 4(n+1), \ldots, 4(n+1)), \\
& \mathbf{H}_{\mathbf{3}}=\operatorname{diag}(2(n+1), 2(n+1), \ldots, 2(n+1)) .
\end{aligned}
$$

Hence, we find that $\mathbf{H}(\mathbf{F})$ has the following eigenvalues $\lambda_{11}=0, \lambda_{22}=n+3, \lambda_{33}=\cdots=$ $\lambda_{n n}=2(n+1), \lambda_{i j}=4(n+1), \lambda_{i n}=2(n+1), \forall i, j \in\{1,2, \ldots, n-1\}, i \neq j$. Therefore, we deduce that $\mathbf{F}$ is parabolic and reaches a minimum $\mathbf{F}\left(h^{c}\right)$ for the solution $h^{c}$ of (6.4-6.7). In fact, because of the convexity, the critical point $h^{c}$ is a global minimum. But we obtain $\mathbf{F}\left(h^{c}\right)=0$. Thus, we deduce $\mathbf{F} \geq 0$. It is easy to derive the following inequality:

$$
\rho \leq \delta_{\mathbf{C}}^{\prime}(n-1)+\left(\alpha^{2}+\beta^{2}-\delta \xi \beta\right)-\frac{1}{n}[(\delta)(n-2)+2 \beta] .
$$

The equality case of (6.9) holds if and only if we have the equality in all the previous inequalities and we find

$$
\begin{aligned}
& h_{i j}^{a}=0, \quad i \neq j, \quad \forall a, \\
& h_{n n}^{a}=2 h_{11}^{a}=2 h_{22}^{a}=\cdots=2 h_{n-1, n-1}^{a}, \quad \forall a .
\end{aligned}
$$
(6.2):

Thus, we state the following main theorem for the normalized $\delta^{\prime}$-Casorati curvature $\delta_{\mathbf{C}}^{\prime}(n-1)$

Theorem 6.1. Let $\mathbf{M}$ be an $n$-dimensional, $n>2$, submanifold of an $m$-dimensional $\delta$-Lorentzian trans-Sasakian manifold $\overline{\mathbf{M}}$ with a SSM connection and whose curvature tensor is of the form (3.2). Then the normalized $\delta^{\prime}$-Casorati curvature $\delta_{\mathbf{C}}^{\prime}(n-1)$ satisfies

$$
\rho \leq \delta_{\mathbf{C}}^{\prime}(n-1)+\left(\alpha^{2}+\beta^{2}-\delta \xi \beta\right)-\frac{1}{n}[(\delta)(n-2)+2 \beta] .
$$

Moreover, the equality case holds if and only if $\mathbf{M}^{n}$ is an invariantly quasi-umbilical submanifold with trivial normal connection in $\overline{\mathbf{M}}^{m}$, such that with respect to suitable orthonormal tangent frame $\left\{e_{1}, \ldots, e_{n}\right\}$ and normal orthonormal frame $\left\{e_{n+1}, \ldots, e_{m}\right\}$, the shape operators $A_{a}=A e_{a}$, $a \in\{n+1, \ldots, m\}$, take the following forms:

$$
A_{n+1}=\left(\begin{array}{cccccc}
t_{1} & 0 & 0 & \ldots & 0 & 0 \\
0 & t_{1} & 0 & \ldots & 0 & 0 \\
0 & 0 & t_{1} & \ldots & 0 & 0 \\
\vdots & \vdots & \vdots & \ddots & \vdots & \vdots \\
0 & 0 & 0 & \ldots & t_{1} & 0 \\
0 & 0 & 0 & \ldots & 0 & 2 t_{1}
\end{array}\right), \quad A_{n+2}=\cdots=A_{m}=0
$$


Similarly, for the normalized $\delta^{\prime}$-Casorati curvature $\hat{\delta}_{\mathbf{C}}^{\prime}(n-1)(6.3)$, we have

Theorem 6.2. Let $\mathbf{M}$ be an n-dimensional, $n>2$, submanifold of an m-dimensional $\delta$-Lorentzian trans-Sasakian manifold $\overline{\mathbf{M}}$ with a SSM connection and whose curvature tensor is of the form (3.2). Then the normalized $\delta^{\prime}$-Casorati curvature $\hat{\delta}_{\mathbf{C}}^{\prime}(n-1)$ satisfies

$$
\rho \leq \hat{\delta}_{\mathbf{C}}^{\prime}(n-1)+\left(\alpha^{2}+\beta^{2}-\delta \xi \beta\right)-\frac{1}{n}[(\delta)(n-2)+2 \beta] .
$$

Moreover, the equality case holds if and only if $\mathbf{M}^{n}$ is an invariantly quasi-umbilical submanifold with trivial normal connection in $\overline{\mathbf{M}}^{m}$, such that with respect to suitable orthonormal tangent frame $\left\{e_{1}, \ldots, e_{n}\right\}$ and normal orthonormal frame $\left\{e_{n+1}, \ldots, e_{m}\right\}$, the shape operators $A_{a}=A e_{a}$, $a \in\{n+1, \ldots, m\}$, take the following forms:

$$
A_{n+1}=2\left(\begin{array}{cccccc}
t_{1} & 0 & 0 & \ldots & 0 & 0 \\
0 & t_{1} & 0 & \ldots & 0 & 0 \\
0 & 0 & t_{1} & \ldots & 0 & 0 \\
\vdots & \vdots & \vdots & \ddots & \vdots & \vdots \\
0 & 0 & 0 & \ldots & t_{1} & 0 \\
0 & 0 & 0 & \ldots & 0 & \frac{t_{1}}{2}
\end{array}\right), \quad A_{n+2}=\cdots=A_{m}=0
$$

As a consequence of Theorem 6.1, we give the following results:

Corollary 6.3. Let $\mathbf{M}$ be an $n$-dimensional, $n>2$, submanifold of an $m$-dimensional of a Lorentzian cosymplectic manifold $\overline{\mathbf{M}}$ of type $(0,0)$ with a SSM connection and whose curvature tensor is of the form (3.2). Then

$$
\rho \leq \delta_{\mathbf{C}}^{\prime}(n-1)-\frac{n-2}{n}(\delta) .
$$

Corollary 6.4. Let $\mathbf{M}$ be an $n$-dimensional, $n>2$, submanifold of an $m$-dimensional of a Lorentzian $\beta$-Kenmotsu manifold $\overline{\mathbf{M}}$ of type $(0, \beta)$ with a SSM connection and whose curvature tensor is of the form (3.2). Then

$$
\rho \leq \delta_{\mathbf{C}}^{\prime}(n-1)+\left(\beta^{2}-\delta \xi \beta\right)-\frac{1}{n}[(\delta)(n-2)+2 \beta] .
$$

Corollary 6.5. Let $\mathbf{M}$ be an $n$-dimensional, $n>2$, submanifold of an $m$-dimensional of a Lorentzian $\alpha$-Sasakina manifold $\overline{\mathbf{M}}$ of type $(\alpha, 0)$ with a SSM connection and whose curvature tensor is of the form (3.2). Then

$$
\rho \leq \delta_{\mathbf{C}}^{\prime}(n-1)+\alpha^{2}-\frac{n-2}{n}(\delta) .
$$

The equality case holds in Corollaries $6.3-6.5$ if and only if $\mathbf{M}^{n}$ is an invariantly quasiumbilical submanifold with trivial normal connection in $\overline{\mathbf{M}}^{m}$, such that with respect to suitable orthonormal tangent frame $\left\{e_{1}, \ldots, e_{n}\right\}$ and normal orthonormal frame $\left\{e_{n+1}, \ldots, e_{m}\right\}$, the shape operators $A_{b}=A e_{b}, b \in\{n+1, \ldots, m\}$, take forms as in 6.11 . 
Similar consequences of Theorem 6.2 can be discussed.

The results, in this section, are given for submanifolds with dimensions higher than 2 . Therefore, we give an example of $\delta$-Lorentzian trans-Sasakiam manifold with dimension 5 .

Example 7. Let us consider a 5-dimensional manifold $\overline{\mathbf{M}}=\left\{\left(x_{1}, x_{2}, x_{3}, x_{4}, z\right) \in \mathbb{R}^{5}: z \neq 0\right\}$, where $\left(x_{1}, x_{2}, x_{3}, x_{4}, z\right)$ are standard coordinates in $\mathbb{R}^{5}$. We choose the vector fields.

$v_{1}=e^{-z} \frac{\partial}{\partial x_{1}}, v_{2}=e^{-z} \frac{\partial}{\partial x_{2}}, v_{3}=e^{-z} \frac{\partial}{\partial x_{3}}, v_{4}=e^{-z} \frac{\partial}{\partial x_{4}}, v_{5}=e^{-z} \frac{\partial}{\partial x_{1}}$, which are linearly independent at each point of $\bar{M}$. We define $g$ by

$$
g=e^{2 z} g
$$

where $g$ is the Lorentzian metric on $R^{5}$ same as in Example 5

$$
g\left(v_{i}, v_{j}\right)=0, \quad i \neq j, i, j=1, \ldots 5 \quad g\left(v_{i}, v_{j}\right)=1, \quad i=j, i, j=1 \ldots 4, \quad g\left(v_{5}, v_{5}\right)=-\delta,
$$

Hence, $\left\{v_{1}, v_{2}, v_{3}, v_{4}, v_{5}\right\}$ is an orthonormal basis of $\overline{\mathbf{M}}$. We consider an 1-form $\eta$ defined by

$$
\eta=e^{z} d z, \quad \eta(X)=\delta g\left(X, v_{5}\right), \quad \forall X \in T \overline{\mathbf{M}} .
$$

Let $\phi$ be a $(1,1)$ tensor field. Thus, we have

$$
\phi\left(v_{1}\right)=v_{3}, \phi\left(v_{2}\right)=v_{4}, \phi\left(v_{3}\right)=-v_{1}, \phi\left(v_{4}\right)=-E_{2}, \phi\left(v_{5}\right)=0 .
$$

The linear property of $g$ and $\phi$ yields that

$$
\eta\left(v_{5}\right)=-1, \quad \phi^{2}(X)=X+\eta(X) v_{5}
$$

and

$$
g(\phi X, \phi Y)=g(X, Y)+\delta \eta(X) \eta(Y),
$$

for any vector fields $X, Y$ on $\overline{\mathbf{M}}$. Thus, $\overline{\mathbf{M}}(\phi, \xi, \eta, g)$ define s a Lorentzian manifold with timelike vector field $\xi=E_{5}$. Moreover, let $\nabla$ be the Levi-Civita connection with respect to metric $g$. Then, we have $\left[v_{1}, v_{2}\right]=0$. Similarly $\left[v_{1}, \xi\right]=e^{-z} v_{1},\left[v_{2}, \xi\right]=e^{-z} v_{2},\left[v_{3}, \xi\right]=e^{-z} v_{3}\left[v_{4}, \xi\right]=$ $e^{-z} v_{4},\left[v_{i}, v_{j}\right]=0,1 \leq i \neq j \leq 4$.

The Riemannian connection $\nabla$ of the metric $g$ is given by

$$
\begin{aligned}
2 g\left(\nabla_{X} Y, Z\right)= & X g(Y, Z)+Y g(Z, X)-Z g(X, Y)-g(X,[Y, Z]) \\
& -g(Y,[X, Z])+g(Z,[X, Y]) .
\end{aligned}
$$

By Koszul's formula, we obtain the following equations:

$$
\begin{gathered}
\nabla_{v_{1}} v_{1}=-e^{-z} \xi, \nabla_{v_{2}} v_{2}=-e^{-z} \xi, \nabla_{v_{3}} v_{3}=-e^{-z} \xi, \nabla_{v_{4}} v_{4}=-e^{-z} \xi \\
\nabla_{\xi} \xi=0, \nabla_{\xi} v_{i}=0, \nabla_{v_{i}} \xi=e^{-z} v_{i}, 1 \leq i \leq 4
\end{gathered}
$$

and $\nabla_{v_{i}} v_{i}=0$ for all $1 \leq i, j \leq 4$. Thus, we see that $\overline{\mathbf{M}}$ is a $\delta$-Lorentzian trans-Sasakian manifold of type $\left(0, e^{-z}\right)$, where $\alpha=0$ and $\beta=e^{-z}$. 


\section{Open Problems}

1. In [16] J.C. Marrero proved that, at the Riemannian case, for dimensions greater or equal to 5 the only existing trans-Sasakian manifolds are Sasakian and Kenmotsu ones. Maybe it is different in the semi-Riemannian case, this would be a really interesting open problem for the geometers.

2. The optimization techniques have a pivotal role in improving inequalities involving Chen invariants. T. Oprea [18, 19, 20] applied the constrained extremum problem to prove Chen-Ricci inequalities for Lagrangian submanifolds of complex space forms. In the characterization of our main result, is it possible to apply the following lemma to derive such inequalities (Theorems 6.1 and 6.2 )?

Let $(\bar{B}, \bar{g})$ be a Riemannian manifold, $B$ be a Riemannian submanifold of it, $g$ be the metric induced on $B$ by $\bar{g}$ and $f:(\bar{B}, \bar{g}) \longrightarrow(\mathbb{R},<\cdot, \cdot>)$ be a differentiable function. Consider the constrained extremum problem $\min _{x \in B} f(x)$, then we have the following:

Lemma 7.1. If $x_{0} \in B$ is the solution of the above problem, then

(a) $(\operatorname{gradf})\left(x_{0}\right) \in T_{x_{0}}^{\perp} B$,

(b) the bilinear form

$$
\mathbf{A}: T_{x_{0}} B \times T_{x_{0}} B \longrightarrow \mathbb{R}, \mathbf{A}(X, Y)=\operatorname{Hess}_{f}(X, Y)+\bar{g}\left(h(X, Y),(\operatorname{gradf})\left(x_{0}\right)\right)
$$

is positive semi-definite, where $h$ is the second fundamental form of $B$ in $\bar{B}$ and gradf denotes gradient of $f$.

If $(\bar{B}, \bar{g})$ is a semi-Riemannian manifold (or particulary a Lorentzian manifold) in Lemma 7.1 , is $\mathbf{A}(X, Y)$ positive semi-definite?

\section{Acknowledgements}

The authors are grateful to the referee for the valuable suggestions and comments towards the improvement of the paper.

\section{References}

[1] S. M. Bhati, On weakly Ricci $\phi$-symmetric $\delta$-Lorentzian trans-Sasakian manifolds, Bull. Math. Anal. Appl., 5 (2013), 36-43.

[2] E. Bartolotti, Sulla geometria della variata a connection affine, Ann. di Mat., 4 (1930), 53-101.

[3] A. Bejancu and K. L. Duggal, Real hypersurfaces of indefinite Kaehler manifolds, Int. J. Math. Math. Sci., 16 (1993), 545-556.

[4] D. E. Blair, Contact manifolds in Riemannian geometry, Lecture note in Mathematics, Springer-Verlag Berlin-New York, 509 (1976).

[5] B.-Y. Chen, Recent developments in $\delta$-Casorati curvature invariants, Turk. J. Math., 45 (2021), 1-46. 
[6] S. Decu, S. Haesen and L. Verstraelen, Optimal inequalities involving Casorati curvatures, Bull. Transilv. Univ. Brasov Ser. B., 14 (2007), 85-93.

[7] A. Friedmann and J. A. Schouten, Uber die Geometric der halbsymmetrischen Ubertragung, Math. Z., 21 (1924), 211-223.

[8] H. Gill and K. K. Dube, Generalized CR- Submanifolds of a trans-Lorentzian para-Sasakian manifold, Proc. Nat. Acad. Sci. India Sec. A Phys., 2 (2006), 119-124.

[9] A. Gray and L. M. Harvella, The sixteen classes of almost Hermitian manifolds and their linear invariants, Ann. Mat. Pura Appl., 123 (1980), 35-58.

[10] H. A. Hayden, Subspaces of space with torsion, Proc. London Math. Soc., 34 (1932), 27-50.

[11] I. E. Hirică and L. Nicolescu, Conformal connections on Lyra manifolds, Balkan J. Geom. Appl., 13 (2008), 43-49.

[12] A. Haseeb, A. Ahamd and M. D. Siddiqi, On contact $C R$-submanifolds of a $\delta$-Lorentzian trans-Sasakian manifold, Global J. Adv. Res. Class. Mod. Geom., 6 (2017), 73-82.

[13] A. Haseeb, M. A. Khan and M. D. Siddiqi, Some results on an $(\varepsilon)$ - Kenmotsu manifolds with a semi-symmetric semi- metric connection, Acta Mathematica Universitatis Comenianae, 85, (2016), 9-20.

[14] J. B. Jun, U. C. De and G. Pathak, On Kenmotsu manifolds, J. Korean Math. Soc., 42 (2005), 435-445.

[15] C. W. Lee, D. W. Yoon and J. W. Lee, Optimal inequalities for the Casorati curvatures of submanifolds of real space forms endowed with semi-symmetric metric connections, J. Inequal. Appl., 2014, 327 (2014).

[16] J. C. Marrero, The local structure of trans-Sasakian manifolds, Annali di Mat. Pura ed Appl., 162 (1992), 77-86.

[17] K. Matsumoto, On Lorentzian paracontact manifolds, Bull. Yamagata Univ. Nat. Science, 2 (1989), 151-156.

[18] T. Oprea, Optimization methods on Riemannian submanifolds, An. Univ. Bucur., Mat., 54 (2005), 127-136.

[19] T. Oprea, Chen's inequality in the Lagrangian case, Colloq. Math., 108 (2007), 163-169.

[20] T. Oprea, Ricci curvature of Lagrangian submanifolds in complex space forms, Math. Inequal. Appl., 13 (2010), 851-858.

[21] J. A. Oubina, New classes of almost contact metric structures, Publ. Math. Debrecen, 32 (1985), 187-193.

[22] G. Pathak and U. C. De, On a semi-symmetric connection in a Kenmotsu manifold, Bull. Calcutta Math. Soc., 94 (2002), 319-324.

[23] S. S. Pujar and V. J. Khairnar, On Lorentzian trans-Sasakian manifold-I, Int. J. Ultra Sciences of Physical Sciences, 23 (2011), 53-66. 
[24] A. Sharfuddin and S. I. Hussain, Semi-symmetric metric connections in almost contact manifolds, Tensor (N.S.), 30 (1976), 133-139.

[25] M. D. Siddiqi, M. Ahmad and J. P. Ojha, CR-Submanifolds of a nearly trans-Hyperbolic Sasakian manifold with a semi-symmetric-non-metric connection, African Diaspora Journal of Math., N.S., 17 (2012), 93-105.

[26] M. D. Siddiqi, A. Haseeb and M. Ahmad, A Note On Generalized Ricci-Recurrent $(\epsilon, \delta)$ trans-Sasakian Manifolds, Palestine J. Math., 4 (2015), 156-163

[27] A. N. Siddiqui, Upper bound inequalities for $\delta$-Casorati curvatures of submanifolds in generalized Sasakian space forms admitting a semi-symmetric metric connection, Inter. Elec. J. Geom., 11 (2018), 57-67.

[28] S. Tanno, The automorphism groups of almost contact Riemannian manifolds, Tohoku Math. J., 21 (1969), 21-38.

[29] K. Yano, On semi-symmetric metric connections, Revue Roumaine De Math. Pures Appl., 15 (1970), 1579-1586.

[30] P. Zhang and L. Zhang, Remarks on inequalities for the Casorati curvatures of slant submanifolds in quaternionic space forms, J. Inequal. App., 2014, 2014:452.

Aliya Naaz Siddiqui Department of Mathematics, Maharishi Markandeshwar Deemed to be University, Mullana, 133207, Ambala-Haryana, India

E-mail: aliyanaazsiddiqui9@gmail.com

Mohd. Danish Siddiqi Department of Mathematics, College of Science, Jazan University, Jazan, Kingdom of Saudi Arabia

E-mail: msiddiqi@jazanu.edu.sa

Mohammad Hasan Shahid Department of Mathematics, Jamia Millia Islamia, New Delhi110025, India

E-mail: mshahid@jmi.ac.in 